\title{
Transcriptomic and metabolomic profiling provide novel insights into fruit development and flesh coloration in Prunus mira Koehne, a special wild peach species
}

Hong Ying ${ }^{1,2+}$, Jian Shi ${ }^{3+}$, Shanshan Zhang ${ }^{1,2}$, Gesang Pingcuo ${ }^{1,2}$, Shuo Wang ${ }^{1,2}$, Fan Zhao ${ }^{1,2}$, Yongning Cui ${ }^{1,2}$ and Xiuli Zeng ${ }^{1,2^{*}}$

\begin{abstract}
Background: Flesh color is one of the most important traits for the commercial value of peach fruit. To unravel the underlying regulatory network in Prunus mira, we performed an integrated analysis of the transcriptome and metabolome of 3 fruit types with various flesh pigmentations (milk-white, yellow and blood) at 3 developmental stages (pit-hardening, cell enlargement and fruit ripening).

Results: Transcriptome analysis showed that an intense transcriptional adjustment is required for the transition from the pit-hardening to the cell enlargement stage. In contrast, few genes were differentially expressed (DEGs) from the cell enlargement to the fruit ripening stage and importantly, the 3 fruits displayed diverse transcriptional activities, indicating that difference in fruit flesh pigmentations mainly occurred during the ripening stage. We further investigated the DEGs between pairs of fruit types during the ripening stage and identified 563 DEGs representing the 'core transcriptome' associated with major differentiations between the 3 fruit types, including flesh pigmentation. Meanwhile, we analyzed the metabolome, particularly, at the ripening stage and uncovered 40 differential metabolites ('core metabolome') between the 3 fruit types including 5 anthocyanins, which may be the key molecules associated with flesh coloration. Finally, we constructed the regulatory network depicting the interactions between anthocyanins and important transcripts involved in fruit flesh coloration.

Conclusions: The major metabolites and transcripts involved in fruit flesh coloration in P. mira were unraveled in this study providing valuable information which will undoubtedly assist in breeding towards improved fruit quality in peach.
\end{abstract}

Keywords: Gene regulatory network, Metabolites, Flesh pigmentation, Prunus mira

\footnotetext{
* Correspondence: zeng_xiuli2008@aliyun.com

${ }^{\dagger}$ Hong Ying and Jian Shi contributed equally to this work.

${ }^{1}$ The ministry of agriculture of Qinghai-Tibet plateau fruit trees scientific

observation test station, Lhasa 850032, Tibet, China

${ }^{2}$ Institute of Vegetables, Tibet Academy of Agricultural and Animal

Husbandry Sciences, Lhasa 850002, Tibet, China

Full list of author information is available at the end of the article
}

(c) The Author(s). 2019 Open Access This article is distributed under the terms of the Creative Commons Attribution 4.0 International License (http://creativecommons.org/licenses/by/4.0/), which permits unrestricted use, distribution, and reproduction in any medium, provided you give appropriate credit to the original author(s) and the source, provide a link to the Creative Commons license, and indicate if changes were made. The Creative Commons Public Domain Dedication waiver (http://creativecommons.org/publicdomain/zero/1.0/) applies to the data made available in this article, unless otherwise stated. 


\section{Background}

Prunus mira Koehne (syn. Amygdalus mira) is an important wild peach species native to China and widely distributed in the Tibetan Plateau [1]. It is a perennial fruit tree belonging to the family Rosaceae and the genus Prunus. In China, P. mira has special nutritional, economic, medicinal and ornamental values [2]. Its edible fruit is rich in nutrients (vitamin $\mathrm{C}$, calcium, and iron) and fatty acids (oleic acid, linoleic acid, cetylic acid, and octadecanoic acid). Therefore, $P$. mira fruit and the processed fruit juice are commercialized, generating considerable economic profits [3]. Its fruit is also greatly employed in Chinese traditional medicine to treat irregular menstruations, fractures, and congestion owing to its high potential to enhance blood circulation [1]. The high content of arbutin in the fruit also promotes its use in the skin care industry. Moreover, $P$. mira flowers are recognized as presenting an ornamental interest [4]. As an ancestral species of many cultivated peach species including Prunus persica, P. mira represents a valuable reservoir of useful alleles for peach improvement [5]. At present, there are few systematic studies on the characteristics and quality of $P$. mira fruit. In contrast to the research on its cultivation which started very early, molecular research was instigated late, explaining the lack of progress in the exploitation and valorization of $P$. mira germplasm resources in China [6].

Flesh color is one of the most important traits for the commercial value of peach fruit which has implications for consumer acceptance and nutritional quality [7, 8]. There is a rich natural variation in Tibetan $P$. mira fruit flesh color [9]. Knowledge of the genetic basis of characters related to Tibetan $P$. mira fruit quality such as the flesh color will facilitate their manipulation to obtain more attractive and healthier fruit for the consumer [10]. Three main classes of accessory pigments, namely, flavonoids, caretonoids, and betalains have been identified in plants [11]. Flavonoids, particularly anthocyanins, provide a wide range of colors ranging from orange/red to violet/blue. The biosynthetic pathway of anthocyanin has been well-characterized in fruit trees [12]. Various structural genes (phenylalanine ammonia-lyase (PAL), chalcone synthase (CHS), chalcone isomerase ( $\mathrm{CHI})$, flavonone 3-hydroxylase (F3H), dihydroflavonol 4reductase (DFR), anthocyanin synthase (ANS), and UDP-glucose-flavonoid 3-O-glucosyltrasnferase (UFGT) and transcription factors such as MYB, basic helixloophelix (bHLH) and WD40 genes were reported to be key determinant of anthocyanin biosynthesis and accumulation $[13,14]$. Betalains are found only in the order Caryophyllales and substitute for anthocyanins [12]. Concerning the carotenoids, they are a class of terpenoids involved in plant photoprotection and coloration [15]. Depending on the concentration and types, carotenoid-rich plant organs can show a wide spectrum of colors including yellow, orange and red [16]. Previous studies in peach species suggested that the red fruit coloration is determined by the content and composition of anthocyanins while the yellow color is associated to carotenoids [17-21]. In addition, it was demonstrated that pigment (anthocyanin and carotenoid) accumulation is dependent on the fruit developmental stages [18, 21, 22]. In the cultivated peach ( $P$. persica L. Batsch), the high activity of the gene $c c d 4$ leading to a low carotenoid level was pinpointed as determinant for the white-flesh phenotype as opposed to the yellow-flesh one $[18,19]$. By studying the blood-flesh and white-flesh peach, Jiao et al. [20] showed that $P A L$ gene was weakly expressed in the white-flesh peach and proposed that $P A L$ gene may be limiting anthocyanin production, leading to the white-flesh coloration.

In recent years, high-throughput methods have been extensively applied to understand organ coloration in plants. Integrated metabolomic and transcriptomic analyses in plant organs shed light on the relationship between the contents of various secondary metabolites and the corresponding differentially expressed genes [23-25]. Combining transcriptome and metabolome approaches, Wang et al. [25] revealed that the red color fading of 'Red Bartlett' (Pyrus communis) cultivar was closely linked to the reduced anthocyanin biosynthesis, the increased anthocyanin degradation and the suppression of anthocyanin transport. Another important study in fig (Ficus carica L.) reported that the anthocyanin metabolite cyaniding O-malonylhexoside increased 3992-fold in the purple peel cultivar compared to the green peel cultivar, similarly as various other anthocyanin pathway components [26]. By analyzing the gene expression change, they observed that the high accumulation of anthocyanin compounds was correlated with an upregulation of genes encoding flavonoid and anthocyanin pathway components and transcription factors such as R2R3-MYB in the purple peel cultivar mainly at the mature stages. Recently, Li et al. [22] also identified 7 flavonoids metabolites (bracteatin, luteolin, dihydromyricetin, cyanidin, pelargonidin, delphinidin and (-)-epigallocatechingenes) and six genes (AaF3H, AaLDOX, AaUFGT, $A a M Y B, A a b H L H$, and $A a H B 2)$ as the best candidates involved in the pigmentation of all-red-fleshed and allgreen-fleshed in Actinidia arguta.

In the present work, we investigated for the first time $P$. mira fruit flesh coloration in relation to the developmental stages using integrated transcriptomic and metabolomic analyses. Fruit from two wild trees: PMHR (blood-colored flesh), PMHY (yellow-colored flesh) and a semi-wild tree PMHF (milk-white colored flesh), were harvested at the pit-hardening, cell enlargement and fruit ripening stages. This study aimed at assessing the metabolic pathways and candidate genes underlying the 
various flesh colorations. On the other hand, we explored the dynamism of the transcriptome and metabolome changes at different fruit development stages. Our results provide further insights into fruit development and flesh coloration in plants, shed light on the enormous chemical diversity in P. mira fruit and will undoubtedly assist in efforts to improve fruit appeal and quality in peach species.

\section{Results}

Overview of the fruit transcriptome sequencing in $P$. mira Three types of $P$. mira fruit (PMHY, PMHR and PMHF) with various flesh colorations were collected at different development stages: pit hardening (A), cell enlargement (B) and fruit ripening $(C)$ and used to construct nine sequencing libraries named PMHYA, PMHYB, PMHYC, PMHRA, PMHRB, PMHRC, PMHFA, PMHFB and PMHFC (Fig. 1). With 3 biological replicates, transcriptome sequencing of the 27 samples yielded a total of 195.56 Gb clean data with $92.21 \%$ of bases scoring Q30 and above (Additional file 1: Table S1). Of the total clean reads, 75.44 to $84.41 \%$ were unique matches with the $P$. persica reference genome (ftp://ftp.ncbi.nlm.nih.gov/genomes/all/GCF/000/346/465/GCF_000346465.2_Prunus_ persica_NCBIv2/GCF_000346465.2_Prunus_persica_ NCBIv2_genomic.fna.gz). A total of 17,533 expressed genes in PMHFA, 17,725 in PMHFB, 17,248 in PMHFC, 17,524 in PMHRA, 17,719 in PMHRB, 17,531 in PMHRC, 17,291 in PMHYA, 17,671 in PMHYB, 17,740 in PMHYC were detected, resulting in 23,809 unique genes in $P$. mira (Additional file 2: Table S2). Based on the novel gene excavation analysis, a total of 1054 genes were specific to $P$. mira, 864 of which were functionally annotated (Additional file 3: Table S3). However, it is probable that these genes do exist in peach genome and were not detected by the prediction algorithm used for the reference set of genes. In addition 4037 genes were optimized based on the gene structure optimization analysis in P. persica (Additional file 4: Table S4, Additional file 5: Table S5). Hierarchical clustering of the samples based on the number of fragments per kilobase of exon per million fragments mapped (FPKM) showed that all the biological replicates clustered together, suggesting a high reliability of our sequencing data (Fig. 2). Furthermore, it could be observed that global gene expression varied distinctly from one fruit development stage to another but the variation according to the flesh color was insignificant. This result implies that the expression of a large number of genes is altered during fruit development whereas only a few genes are associated with the differences in fruit flesh coloration in P. mira.

\section{Change in gene expression during fruit developmental stages in $P$. mira}

To uncover the main genes differentially expressed over the different fruit development stages and those specific to the transition from the pit hardening to the cell enlargement stages (AvsB) and subsequently to the fruit ripening stage (BvsC), we cross-compared the differential expressed genes (DEGs) in PMHF, PMHY, PMHR and sorted out the shared DEGs (Additional file 6: Table S6). For AvsB, we detected 8080, 7416 and 6976 DEGs in PMHF, PMHY and PMHR, respectively, with 4079 DEGs commonly identified in the three fruit types (Fig. 3a). Regarding BvsC, we identified 2357, 5344 and 5559 DEGs in PMHF, PMHY and PMHR, respectively, of which, only 950 DEGs were common to the 3 fruit types (Fig. 3b). These results indicate that the molecular mechanisms occurring at the pit hardening and cell enlargement stages are quite well conserved among the different fruit types but the fruit ripening process involves differential molecular mechanisms in the various fruit types. Accordingly, the fruit ripening stage should be targeted to get insights into the altered pathways and genes associated with the fruit flesh coloration in $P$. mira. As shown in Fig. 3c, 343 genes (enriched in KEGG pathways related to MAPK signaling pathway, starch and sucrose metabolism, steroid biosynthesis, carotenoid biosynthesis, etc. (Additional file 7: Figure S1)), were constantly and differentially expressed during the transitions from $\mathrm{A}$ to $\mathrm{B}$ and $\mathrm{B}$ to $\mathrm{C}$, denoting that they

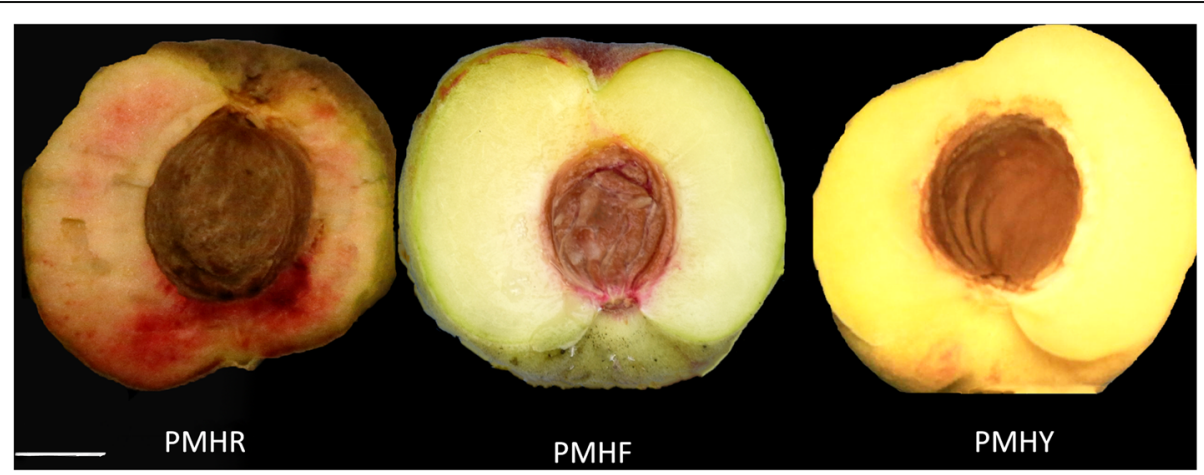

Fig. 1 The phenotypes of the fruit flesh of Prunus mira cv. "PMHR", "PMHF" and "PMHY". Bar $=1 \mathrm{~cm}$ 

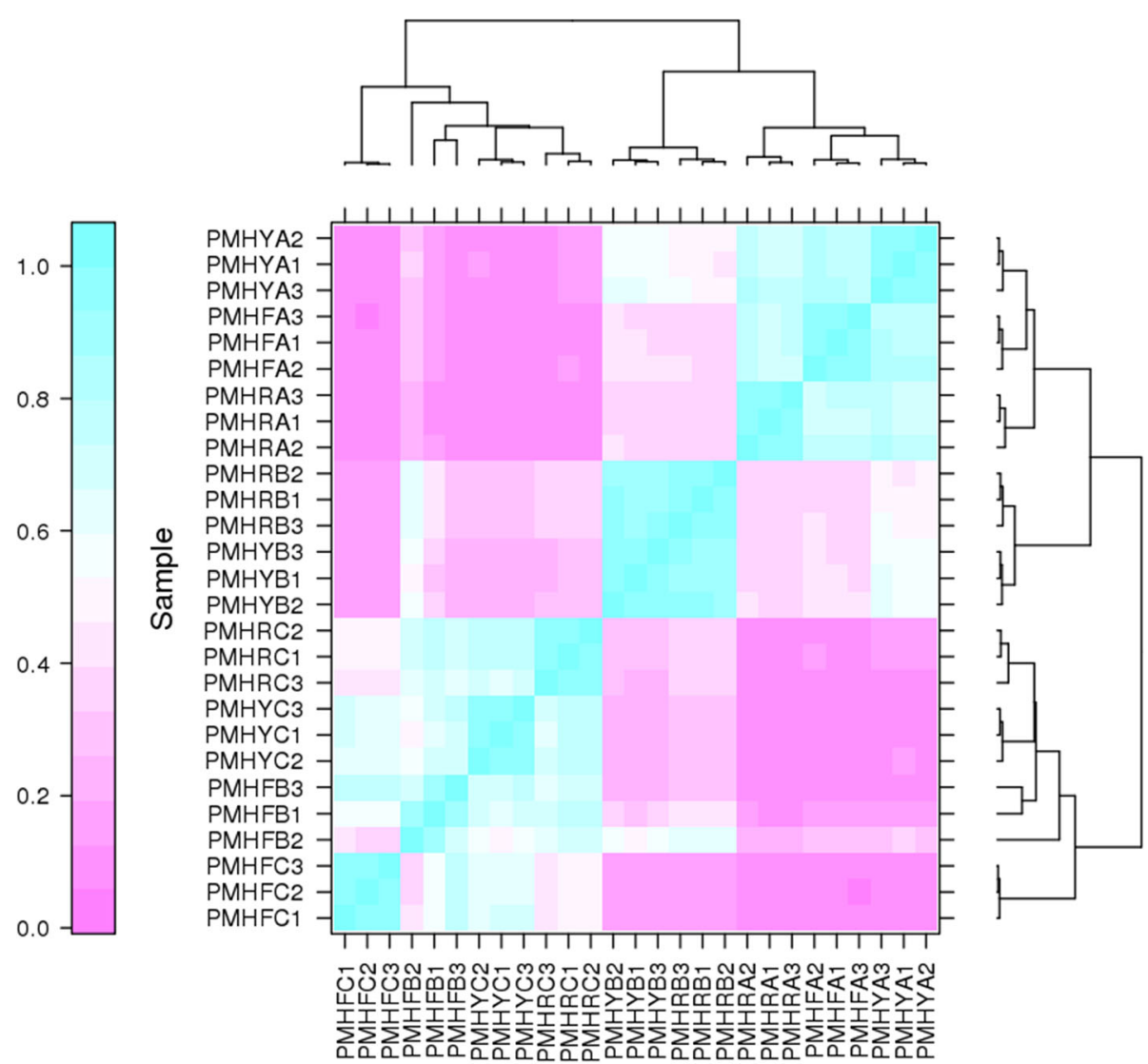

Sample

Fig. 2 Heatmap clustering showing correlation among Prunus mira different samples based on global expression profiles

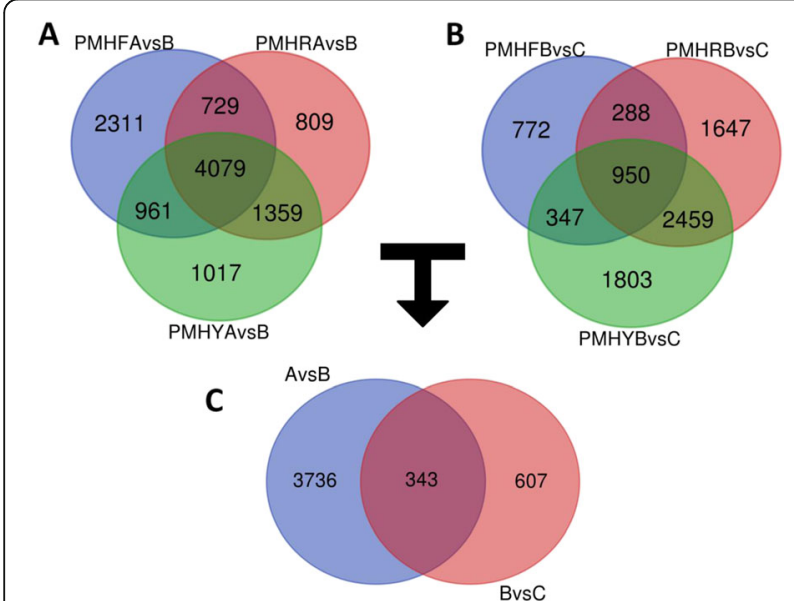

Fig. 3 Differential expressed genes (DEG) during fruit developmental stages in Prunus mira. a. Venn diagram showing the shared and unique DEGs between pit-hardening (A) and cell enlargement (B) stages in PMHF, PMHR and PMHY; $\mathbf{b}$. Venn diagram showing the shared and unique DEGs between cell enlargement (B) and fruit ripening $(C)$ stages in PMHF, PMHR and PMHY; c. Venn diagram showing the shared and unique DEGs between the transitions from $A$ to $B$ and $B$ to $C$ represent the main genes associated to fruit development in P. mira, regardless of the fruit type. Meanwhile, we obtained 3736 genes (enriched in KEGG pathways related to MAPK signaling pathway, amino sugar and nucleotide sugar metabolism, biosynthesis of antibiotics, starch and sucrose metabolism, amino sugar and nucleotide sugar metabolism, etc. (Additional file 8: Figure S2)) and 607 genes (enriched in KEGG pathways related to plant hormone signaling pathways, MAPK signaling pathways, cysteine and methionine metabolism, etc. (Additional file 9: Figure S3)) specific to the transition from $A$ to $B$ and $B$ to $C$, respectively. A total of five genes were randomly selected within the DEGs list and their FPKM fold changes in the three genotypes at the three fruit developmental stages were well correlated with the relative expression via $\mathrm{qRT}-\mathrm{PCR}\left(R^{2}=0.74\right.$; Additional file 10: Figure S4).

\section{Identification of differential expressed genes related to fruit flesh coloration}

To identify the DEGs related to fruit flesh coloration, we compared the FPKM values of PMHF to PMHY (milk white -colored flesh compared to yellow-colored flesh), 
PMHF to PMHR (milk white -colored flesh compared to blood-colored flesh) and PMHR to PMHY (blood-colored flesh compared to yellow-colored flesh) at the fruit ripening stage $(\mathrm{C})$. For PMHFvsPMHY, we detected a total of 4296 DEGs including 2094 down- and 2202 upregulated genes. Similarly, we analyzed the DEGs between PMHFvsPMHR and obtained a total of 4523 DEGs including 2387 down- and 2136 up-regulated genes. Concerning PMHRvsPMHY, we found fewer amounts of DEGs (2706) including 1142 down- and 1564 up-regulated genes. We deduced that change from milk-white to blood or yellow coloration may require a larger transcriptional reconfiguration than change from yellow to blood coloration. Next, we compared the DEGs identified in PMHFvsPMHY, PMHFvsPMHR and PMHYvsPMHR to pinpoint those common the three color change schemes. This resulted in 563 genes constitutively and differentially expressed between the 3 fruit types implying that they may represent the 'core transcriptome' associated to major differentiations between the 3 fruit types, including flesh coloration (Fig. 4, Additional file 11: Table S7). They were enriched in KEGG pathways related to plant hormone signal transduction, pentose and glucuronate interconversions, starch and sucrose metabolism, metabolism of xenobiotics by cytochrome P450, etc. (Additional file 12: Figure S5). Within these genes, only 6 DEGs (gene16639, gene14633, gene4519, gene9018, gene18806 and gene24803) are involved in the terpernoid-carotenoid pathways while many genes (gene17724, gene 788 , gene22193, gene13700, gene23130, Prunus_persica_newGene_904, gene23924, gene19650, gene26, gene14431, gene22560, gene 7875 ,

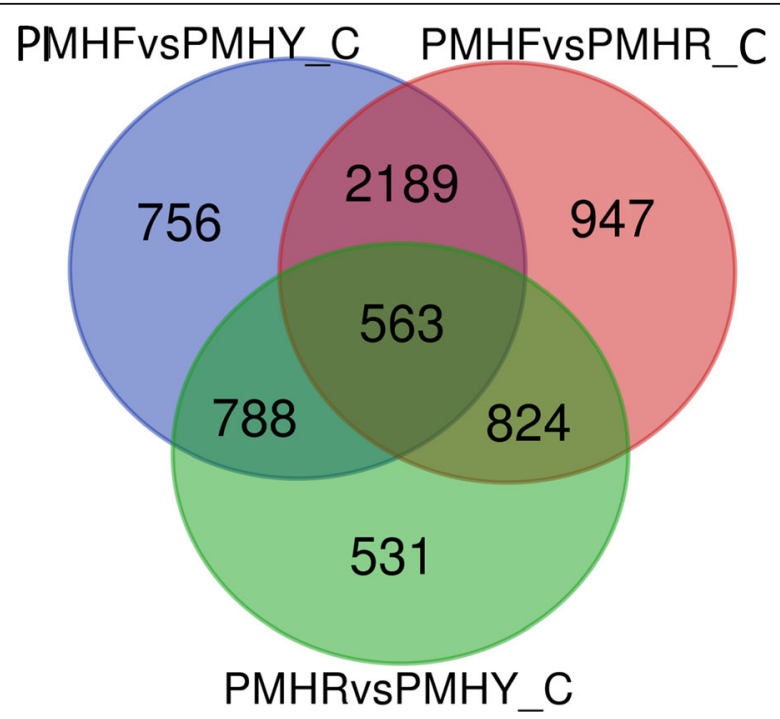

Fig. 4 Identification of the shared and unique differential expressed genes between pairs of fruit types (PMHFvsPMHR, PMHFvsPMHR, PMHRvsPMHY) at the fruit ripening stage (C) in Prunus mira gene4823 and gene21971) participate in the phenylpropanoid-flavonoid pathways (Fig. 5, Additional file 13: Table S8), suggesting that structural genes in the phenylpropanoid-flavonoid pathways are the main regulators of fruit flesh pigmentation in P. mira. In addition, analysis of the transcription factor families including MYB, basic-Helix-Loop-Helix (bHLH) and WD40 which modulate the expression level of flavonoid biosynthetic structural genes unraveled 5 MYBs (gene20317, gene9054, gene25173, gene 13863 and gene22870), 3 bHLHs (gene17547, gene19413 and gene6656) but no WD40 (Additional file 14: Table S9). Within the MYB regulators, 4 genes (gene20317, gene 9054 , gene 25173 and gene22870) were highly expressed in colored flesh fruits (PMHR and PMHY) than PMHF while only the gene gene13863 showed the opposite trend. Similarly, concerning the bHLH transcription factors, only the gene gene19413 was more expressed in PMHF compared to PMHR and PMHY. A total of five genes were randomly selected within the DEGs list and their FPKM fold changes in the three genotypes at the three fruit developmental stages were well correlated with the relative expression via qRT-PCR $\left(R^{2}=0.74\right.$; Additional file 10: Figure S4).

\section{Overview of the fruit flesh metabolome profiling in $P$. mira}

We further analyzed the metabolic alteration in $P$. mira fruit during the 3 developmental stages using a widelytargeted approach. In total, 493 compounds were successfully detected and determined for the first time in $P$. mira (Additional file 15: Table S10). The diversity and richness of the metabolites detected in this work may represent an excellent opportunity to identify novel compounds associated with fruit development, coloration and quality. The identified compounds could be classified into 33 classes, predominantly, organic acids, nucleotide and its derivatives, and amino acid derivatives (Table 1). Principal component analysis (PCA) of the metabolic quantification from the 3 fruit types showed that all biological replicates were grouped together, which indicates a good correlation between replicates and the high reliability of our data. Moreover, we observed that at each stage (A, B, and C), the metabolites from PMHF were obviously distinct from those of PMHR and PMHY (Fig. 6). A separation between the developmental stages could be observed by the PC1 while a separation according to the fruit types by the PC2 could be observed. Overall, these results are consistent with the transcriptional responses between the 3 fruit types.

\section{Analysis of differentially altered metabolites in relation to fruit flesh coloration}

Since the fruit ripening stage was identified as the key period for flesh color determination in P. mira based on the transcriptome results, we scrutinized the 


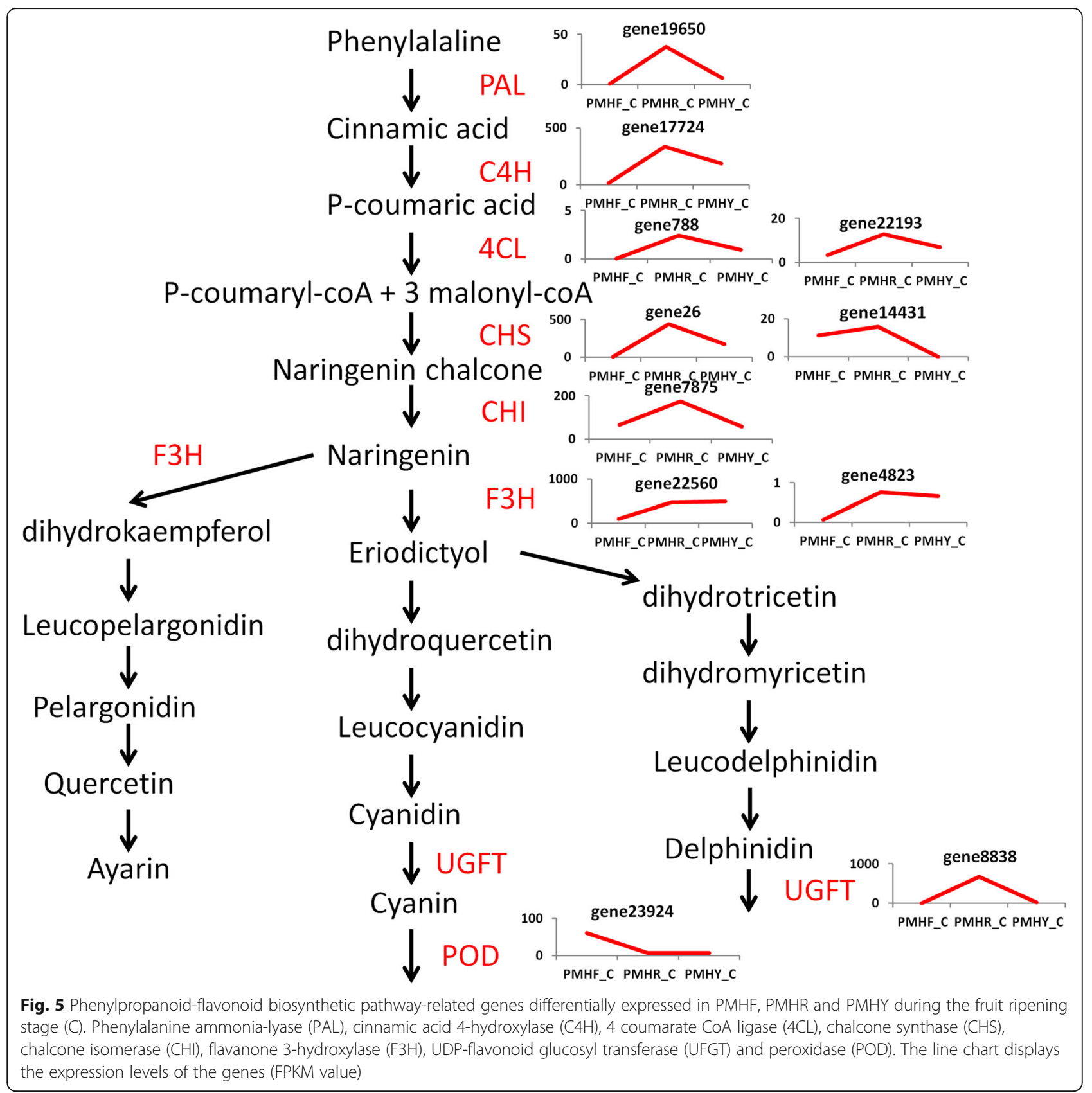

differentially accumulated metabolites (DAM) during this fruit developmental stage in PMHF compared to PMHY (PMHFvsPMHY), PMHF compared to PMHR (PMHFvsPMHR) and PMHR compared to PMHY (PMHRvsPMHY). The differential metabolites between group comparisons were identified based on the foldchange and the variable importance in projection (VIP).

For PMHFvsPMHY, we found 117 DAMs of which, 43 and 74 were down- and up-accumulated compounds, respectively (Fig. 7a). Similarly, for PMHFvsPMHR, a total of 107 DAMs including 50 down- and 57 upaccumulated metabolites were detected (Fig. 7b). We identified 89 DAMs including 27 down- and 62 upaccumulated compounds in PMHRvsPMHY (Fig. 7c). As shown in Fig. 7d, 40 diverse metabolites were commonly shared by the 3 color change schemes, which may constitute the 'core metabolome' associated to major differentiations between the 3 fruit types of $P$. mira (Additional file 16: Table S11). Within these compounds, 25 were mapped to the phenylpropanoid-flavonoid pathways (Fig. 8) and 5 anthocyanins (Jur80, Jur487, Jur861, Jur1147 and Jur929) were detected which may potentially be the key molecules modulating fruit flesh pigmentation in P. mira (Table 2). 
Table 1 Classification of the 493 detected metabolites into major classes

\begin{tabular}{|c|c|c|c|c|c|}
\hline Class & $\begin{array}{l}\text { Number of } \\
\text { compound }\end{array}$ & Class & $\begin{array}{l}\text { Number of } \\
\text { compound }\end{array}$ & Class & $\begin{array}{l}\text { Number of } \\
\text { compound }\end{array}$ \\
\hline Flavonolignan & 1 & Alcohols and polyols & 7 & Carbohydrates & 18 \\
\hline Pyridine derivatives & 1 & Flavone C-glycosides & 7 & Quinate and its derivatives & 20 \\
\hline Terpenoids & 1 & Phenolamides & 7 & Flavone & 21 \\
\hline Isoflavone & 2 & Catechin derivatives & 9 & Flavonol & 22 \\
\hline Alkaloids & 3 & $\begin{array}{l}\text { Benzoic acid } \\
\text { derivatives }\end{array}$ & 10 & Amino acids & 26 \\
\hline $\begin{array}{l}\text { Nicotinic acid } \\
\text { derivatives }\end{array}$ & 3 & Phytohormones & 11 & Others & 26 \\
\hline Tryptamine derivatives & 3 & Coumarins & 12 & $\begin{array}{l}\text { Hydroxycinnamoyl } \\
\text { derivatives }\end{array}$ & 27 \\
\hline Cholines & 4 & Vitamins & 13 & Lipid_Glycerophospholipid & 28 \\
\hline Proanthocyanidins & 4 & Flavanone & 15 & Amino acid derivatives & 45 \\
\hline Anthocyanins & 5 & Lipid_Glycerolipid & 15 & Nucleotide and its derivates & 47 \\
\hline Indole derivatives & 6 & Lipid_Fatty acids & 16 & Organic acids & 58 \\
\hline
\end{tabular}

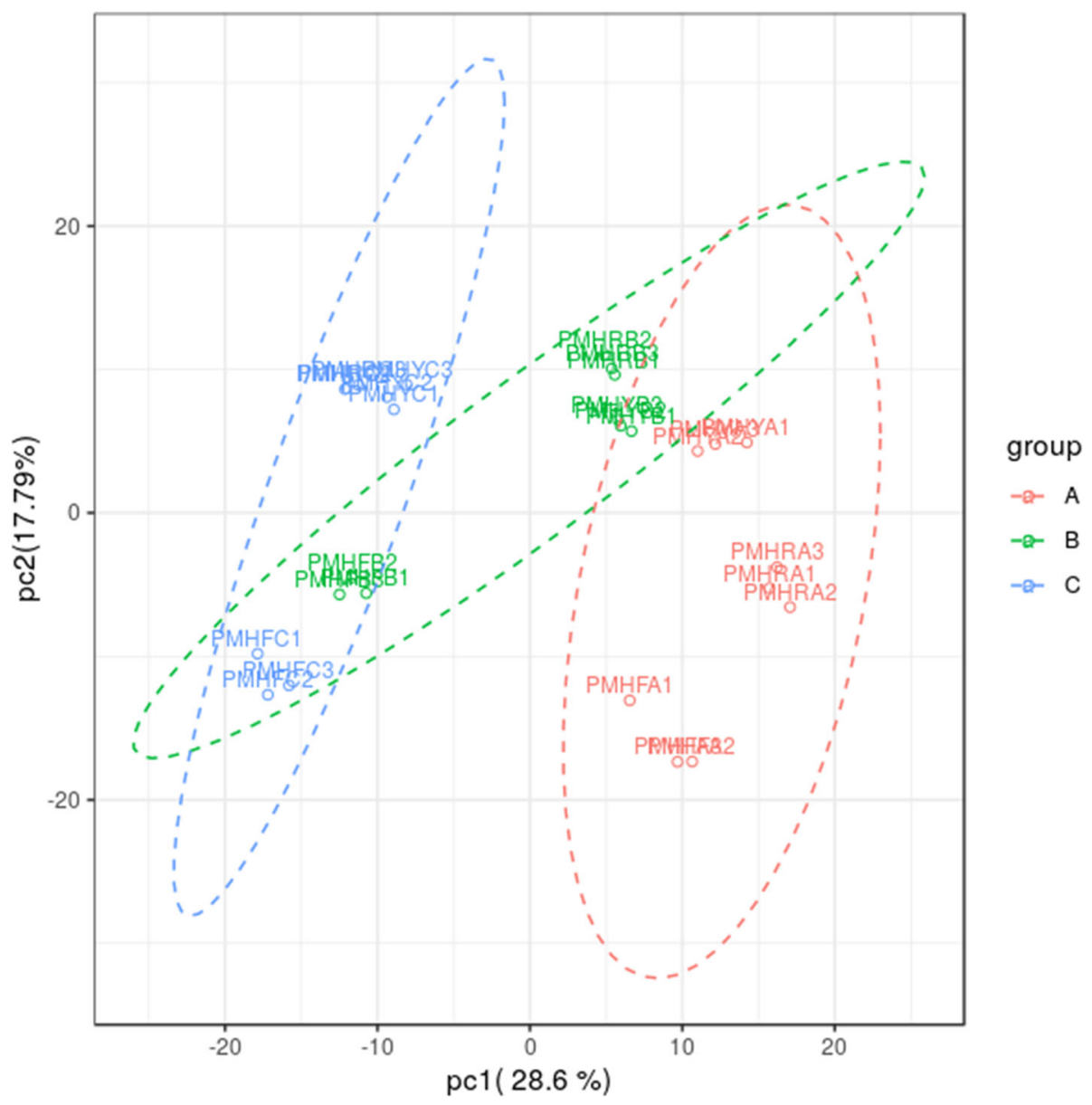

Fig. 6 Principal component analysis of the metabolite quantification in 3 Prunus mira fruit types (PMHF, PMHR and PMHY) at 3 fruit developmental stages (pit-hardening (A), cell enlargement (B) and fruit ripening (C)) 


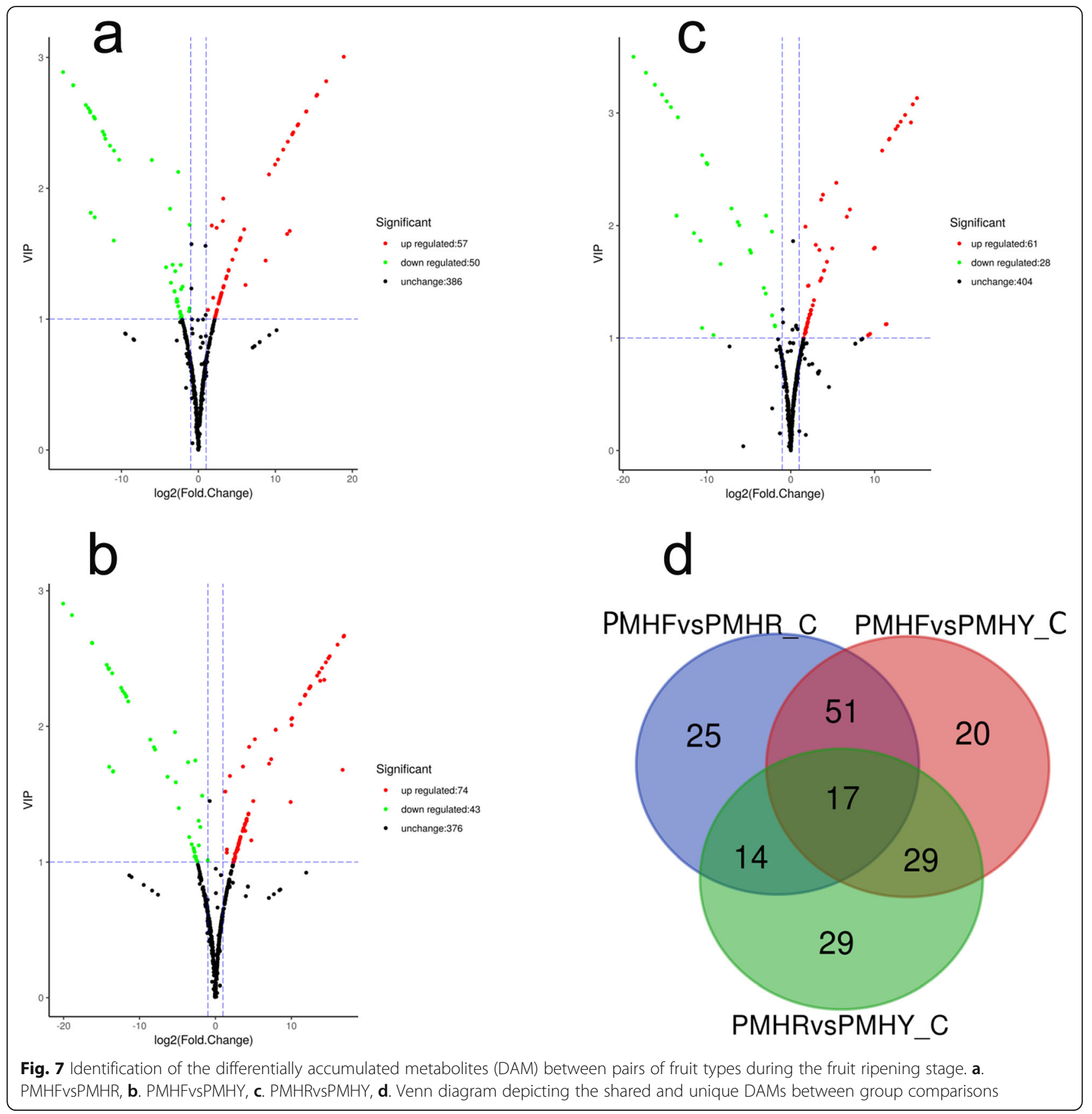

\section{Regulatory network depicting interactions between} important anthocyanins and transcripts associated with fruit flesh coloration in $P$. mira

To understand the regulatory network of anthocyanins involved in the differential pigmentation of the 3 colored $P$. mira fruit, we carried out correlation tests between quantitative changes of the 5 anthocyanins from the 'core metabolome' and transcripts from the 'core transcriptome'. The result showed that 183, 328, 213, 228 and 159 transcripts had strong correlation values $\left(R^{2}>\right.$ 0.9) with Jur487, Jur861, Jur1147, Jur929 and Jur80, respectively (Additional file 17: Table S12). The metabolites Jur80, Jur929 and Jur487 appeared to be more closely related than the other compounds and also shared many similar associated transcripts (Fig. 9). The regulatory network highlighted the major transcripts associated with the anthocyanins and more importantly, the key anthocyanin compounds relevant to each flesh color type. For example, Jur861 (peonidin Omalonylhexoside) was highly accumulated in PMHF while only traces of this metabolite could be detected in PMHR and PMHY, suggesting that Jur861 is important 


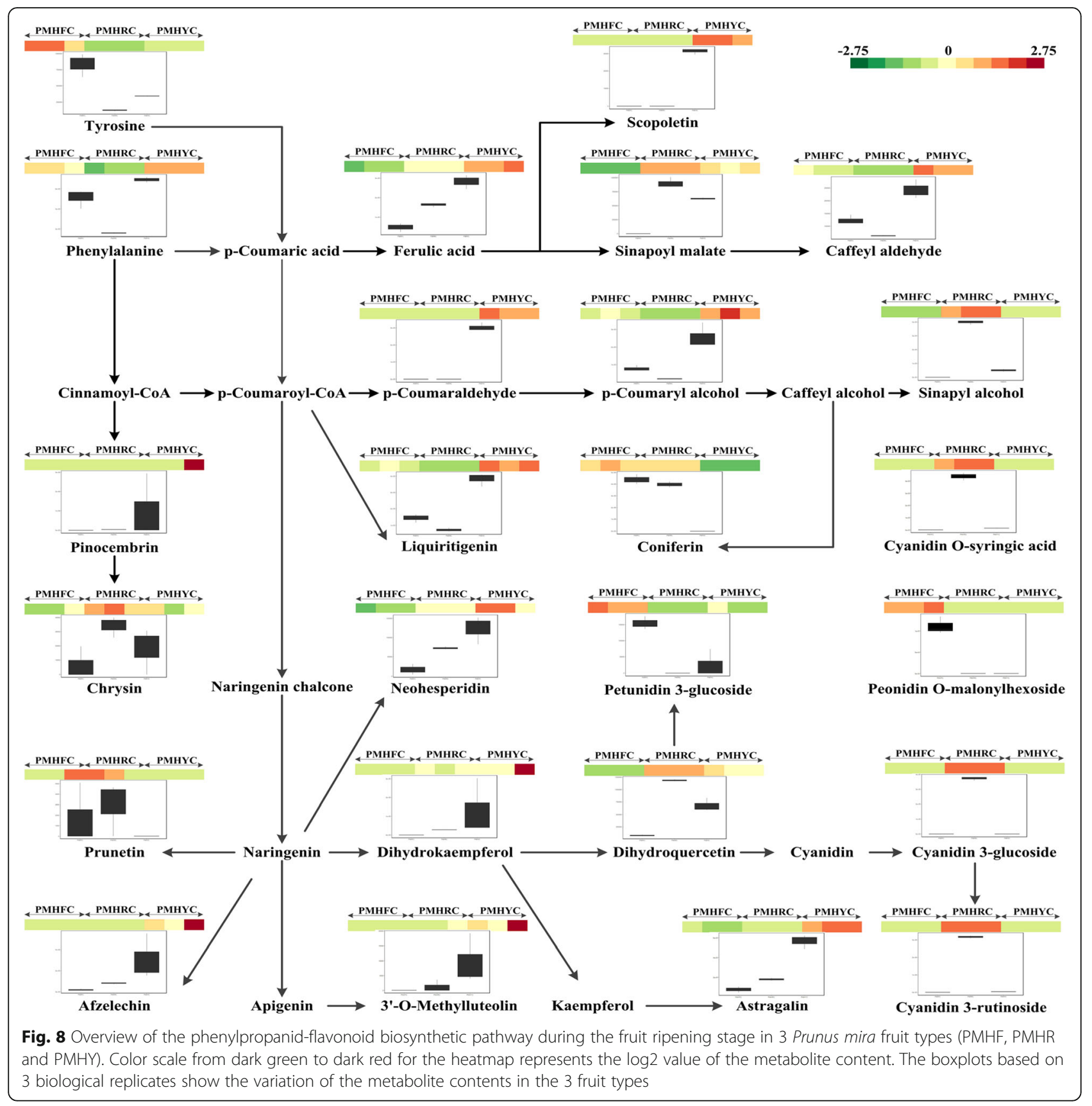

Table 2 List of the anthocyanins identified in the 'core metabolome' regulating flesh coloration in the 3 fruit types and their Log fold change values

\begin{tabular}{llllll}
\hline Compound & Name & Class & PMHF vs PMHR & PMHF vs PMHY & PMHR vs PMHY \\
\hline Jur80 & Cyanidin 3-O-rutinoside (Keracyanin) & Anthocyanins & 9.17 & 3.04 & -6.13 \\
Jur487 & Petunidin 3-O-glucoside & Anthocyanins & -14.06 & -2.64 & 11.42 \\
Jur1147 & Cyanidin O-syringic acid & Anthocyanins & 18.89 & 14.03 & -4.86 \\
Jur929 & Cyanidin 3-O-glucoside (Kuromanin) & Anthocyanins & 15.34 & 2 & -13.34 \\
Jur861 & Peonidin O-malonylhexoside & Anthocyanins & -13.59 & -11.59 & -2 \\
\hline
\end{tabular}




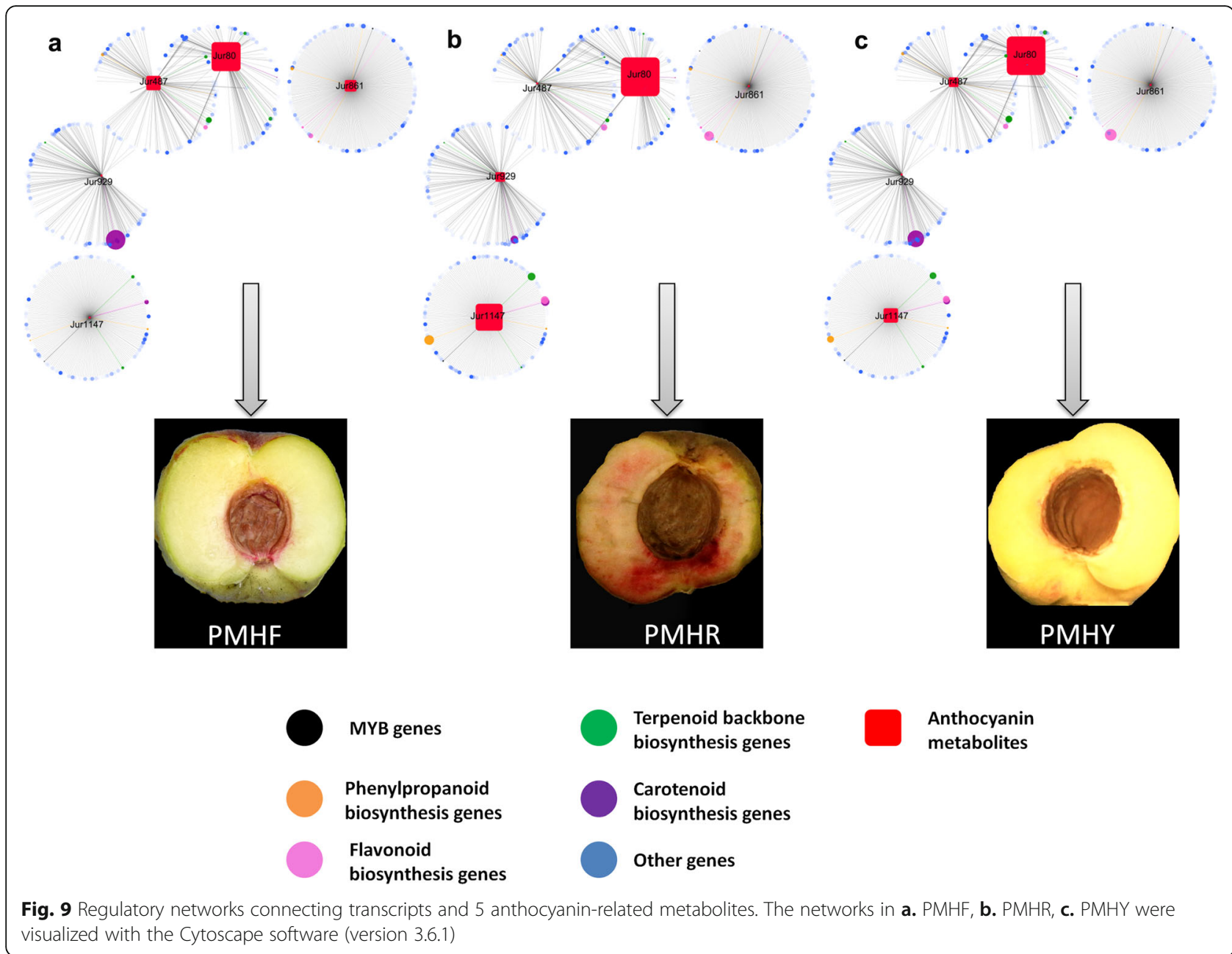

for the milk-white flesh coloration. By analyzing the associated transcripts, the gene gene22560 (naringenin 3dioxygenase) had the highest $R^{2}$ score (0.98) and was upregulated in PMHR and PMHY compared to PMHF ( $\log _{2-}$ foldchange values $=2.39,2.46$, respectively). This indicates that a weak expression of gene 22560 may partly promote peonidin O-malonylhexoside over-accumulation which is associated with the milk white -colored flesh fruit type in $P$. mira. With regard to the compound Jur929 (cyanidin 3-Oglucoside (kuromanin)), it was strongly accumulated in PMHR whereas, only traces could be detected in PMHF and PMHR, indicating that kuromanin is a key molecule for the blood pigmentation in $P$. mira fruit flesh. The gene gene 9018 (phytoene synthase) had the highest correlation score (0.98) with Jur929 but its expression level was significantly low in PMHR compared to PMHY and PMHF $\left(\log _{2}\right.$ fold change values $=1.05,1.07$, respectively). This result implies that gene 9018 may be involved in a negative regulation of kuromanin synthesis in $P$. mira fruit. The compound Jur1147 (cyanidin O-syringic acid) is only present in the PMHR and PMHY but in a higher quantity in PMHR. From the regulatory network, 4 genes (gene24803 ((+)-abscisic acid 8'-hydroxylase), gene17724 (trans-cinnamate 4-monooxygenase), gene21971 (beta-glucosidase) and gene26 (chalcone synthase)) were remarkably important for the accumulation of Jur1147. Analysis of their expression levels showed that they are positively associated with the accumulation of Jur1147 and their higher activities in PMHR could partly explain the higher content of Jur1147. Concerning the metabolite Jur487 (petunidin 3O-glucoside), it was only detected in the fruit flesh of PMHF and PMHY, though, at a lesser quantity in PMHY. Two genes gene7875 (chalcone isomerase) and gene16639 (1-deoxy-D-xylulose-5-phosphate reductoisomerase) from the regulatory network showed high correlation scores with Jur487. The gene gene 7875 was negatively associated with the accumulation of Jur487 while gene16639 showed the opposite trend. Meanwhile, the only metabolite accumulated in a high quantity in all the 3 fruit types was Jur80 (cyanidin 3-O-rutinoside (keracyanin)). However, the exact quantification of keracyanin in the 3 fruit types showed that Jur80 was more accumulated in PMHR $\left(3.17 \mathrm{E}^{+08}\right)$ than 
PMHY $\left(4.52 \mathrm{E}^{+06}\right)$ and PMHF $\left(5.49 \mathrm{E}^{+05}\right)$, denoting that abundant accumulation of Jur80 tends to darken the fruit flesh color, from white to blood.

Overall, the blood coloration may be a combination of a high content of Jur80, Jur1147 and Jur929; the yellow color seemed to result from a moderate accumulation of Jur80, Jur1147 and Jur487; while the milk-white color could be formed thanks to the weak accumulation of Jur80 and a high content of Jur487 and Jur861 in P. mira fruit flesh.

\section{Discussion}

In the present study, an integrated analysis of the transcriptome and metabolome was conducted to elucidate the fruit flesh coloration in Prunus mira, which is an important quality trait in peach [8]. By using the reference genome of Prunus persica [27], we observed a high conservation of the gene models in both species, although 1054 genes were found specific to $P$. mira. These specific genes may represent important wild resources for the improvement of the cultivated peach towards abiotic stress tolerance, fruit quality, disease resistance, etc. [28]. We also provide here for the first time extensive metabolome datasets from P. mira fruit which could fuel future studies on fruit quality traits in wild and cultivated peach species [29].

During the fruit development, the complex network of metabolites and proteins is dramatically altered [30]. Transcriptome and metabolome analyses over 3 fruit developmental stages in $P$. mira indicated that different molecular programs are involved in the transition from the pit-hardening to the cell enlargement stage and subsequently from the cell enlargement to the ripening stage. Previously, Lombardo et al. [29] also reported similar findings in the cultivated peach. They showed that high levels of bioactive polyphenols and amino acids, which are substrates of phenylpropanoid and lignin pathways, are important during pit hardening while a decrease in amino acid levels and an induction of transcripts encoding sucrose degradative and synthetic enzymes, are involved in ripening. In-depth analyses of the specific genes and metabolites detected for each fruit developmental stage will illuminate the biological processes underlying fruit growth and ripening in $P$. mira.

By comparing the transcriptional activity between fruit developmental stages in the 3 fruit types, we observed that they diverged principally during the ripening stage, denoting that major differences in the fruit phenotypes such as flesh pigmentation occur during this stage. Consistent with our report, Roca et al. [31] showed that carotenoids which are involved in plant pigmentation were found differentially accumulated only during ripening in different olive varieties. Furthermore, by analyzing peach fruit for their anthocyanin contents (which are also important for fruit color formation), Cao et al. [32] observed that the flesh anthocyanin contents were mainly differentially accumulated at the early ripening stage in various colored fruit types.

Flavonoids and caretonoids represent the major molecules involved in pigmentation in plants [11]. In this work, flavonoids, particularly, anthocyanins appeared to be more important in $P$. mira fruit flesh pigmentation than carotenoids. This was underscored by the fact that a high number of transcripts belonging to the phenylpropanoid-flavonoid pathways in the 'core transcriptome' and the majority of metabolites detected in the 'core metabolome' were mapped to the phenylpropanoid-flavonoid pathways. The anthocyanin biosynthetic pathway is a specific branch of the flavonoid pathway, which starts from the key amino acid phenylalanine to produce 4-coumaroyl $\mathrm{CoA}$ by phenylalanine ammonia-lyase (PAL), cinnamic acid 4-hydroxylase $(\mathrm{C} 4 \mathrm{H})$ and 4 coumarate CoA ligase (4CL) [33]. The main precursors for flavonoids are 4-coumaroyl $\mathrm{CoA}$ and three molecules of malonyl CoA that produce chalcones by chalcone synthase (CHS) [34]. Then, the pathway is catalyzed by a number of enzymes to yield flavanones (via chalcone isomerase, $\mathrm{CHI}$ ), dihydroflavonols (via flavanone 3-hydroxylase, F3H), leucoanthocyanidins (via dihydroflavonol 4-reductase, DFR), anthocyanidins (via leucoanthocyanidin dioxygenase, LDOX) and anthocyanins (via UDP-flavonoid glucosyl transferase, UFGT) [35]. Previous studies on $P$. persica identified some key structural genes from the flavonoid pathways regulating coloration. The gene $\mathrm{CHS}$ (chalcone synthase, ppa006888m) was pinpointed as an important modulator of the anthocyanin content in P. persica [32, 36]. Here, we found that its ortholog gene26 may also be involved in the modulation of the flavonoid/anthocyanin contents in $P$. mira. In addition to gene26, another CHS gene (gene14431) was also detected but its expression level was lower as compared to gene26. Zhang et al. [37], by investigating the differentially expressed genes controlling the anthocyanin levels in various pigmented nectarines, observed that ppa025745m which is the ortholog of gene14431 was the main candidate gene. We further identified the gene CHI (gene7875) which is highly expressed in the blood-flesh fruit type with 2.6 and 3 -fold increase in expression level compared to the milkwhite and yellow flesh fruit types, respectively. In agreement with our findings, the gene ppa011276m from $P$. persica which is the ortholog of gene7875, was proposed as an important structural gene regulating the red pigmentation in peach flower [38]. Moreover, the gene UFGT (gene8838) which is the ortholog of ppa005162m in P. persica and previously demonstrated to differentially regulate the anthocyanin accumulation in deeply colored and light-pigmented peach cultivars was also revealed in this study. Importantly, ppa005162 $m$ was more expressed in the deeply colored cultivar than in the light pigmented one [32, 39], similarly as gene 8838 which displayed an increase in its expression level 
by 6752- and 35- fold in PMFR and PMHY, respectively, compared to PMHF. Our result perfectly matches the conclusions of Wu et al. [40] who proposed UFGT as the key gene for the red color in Prumus mume flower. Other important flavonoid biosynthetic structural DEGs detected in this study and which may affect the anthocyanin levels in $P$. mira are PAL (gene19650), F3H (gene4823 and gene22560), C4H (gene17724) and 4CL (gene788 and gene22193). Their orthologs were also found to regulate pigmentation in $P$. persica [20,32, 38]. Overall, most of these identified structural genes involved in the regulation of the flesh coloration in P. mira are mapped to early committed steps on the flavonoid biosynthetic pathway and interestingly, they were all highly expressed in the blood flesh fruit, moderately in the yellow flesh fruit and very low in the milk-white flesh fruit, implying that the high activity of these genes lead to a higher anthocyanin content, thus, to a darker pigmentation. Our results corroborate well the conclusions of Chen et al. [38] who also reported that low expression levels of $\mathrm{C} 4 \mathrm{H}, \mathrm{CHS}$ and $\mathrm{F} 3 \mathrm{H}$ in white petals, contrarily to the red petals of peach, reduce the formation of dihydro-kaempferol (DHK), and thereby inhibit the anthocyanin accumulation. In the same line, Jiao et al. [20] also showed that the $P A L$ gene was weakly expressed in the white-flesh peach and suggested that the $P A L$ gene may be limiting in anthocyanin production, resulting in the white-flesh coloration.

The activity of the structural genes in the flavonoid biosynthetic pathway is regulated by other genes such as transcription factors from the families of MYB, bHLH and WD40 [41]. Here, we identified 5 MYB and 3 bHLH genes that may be the central regulators of the expression of structural genes in the flavonoid biosynthetic pathway, leading to differential accumulation of anthocyanins. Some orthologs (ppa010277m, ppa017136m and ppa006295m) of some of these genes in P. persica were previously confirmed to play similar function $[38,39]$. Another important finding from this study is the gene gene23924, the ortholog of ppa007748m in $P$. persica, which encodes a peroxidase (POD) enzyme. The gene gene23924 is the only differentially expressed gene between the 3 fruit types from the phenylpropanoid pathway which was up-regulated in PMHF ( 10-fold increase) compared to PMHR and PMHY. Several studies have demonstrated that POD is involved in the anthocyanin degradation in various plant species such as litchi, grape, plum, Brunfelsia calycina [42-45]. Hence, our result implies that besides the down-regulation of many structural genes in the phenylpropanoid-flavonoid pathways caused by some MYB and bHLH transcription factors, the degradation of anthocyanins through the high activity of gene 23924 could also be another important mechanism for the milk-white coloration obtained in PMHF.

Our metabolic profiling highlighted five key anthocyanins involved in the differential flesh coloration in $P$. mira fruit. The combinations and levels of accumulation of these compounds may confer the visible colorations. In particular, we observed that PMHR strongly accumulated various anthocyanins, PMHY had moderate contents of anthocyanins while PMHF contained low concentration of anthocyanins, which correlated well with the expression levels of the key structural genes from the flavonoid pathways in these three fruit types. We also delineated in this study the regulatory network underlying the accumulation of the 5 major anthocyanins involved in fruit flesh coloration in P. mira. These results could be harnessed by researchers by utilizing genetic approaches to clarify the mechanism of anthocyanin regulation [46]. Intriguingly, some transcripts involved in the carotenoid biosynthetic pathway were found highly correlated with the anthocynanin compounds. It is probable that the accumulation of some carotenoid compounds together with the anthocyanins provides the final visible flesh coloration in $P$. mira fruit. For example, PMHY mainly accumulated anthocyanins which were reported to give a red coloration [39, 47], then, how exactly its yellow pigment is formed is unclear. Previously, Brandi et al. [18] and Falchi et al. [19] demonstrated that a high carotenoid level was determinant for the yellow-flesh coloration in peach. Unfortunately, the widely-targeted metabolomics approach adopted in this study did not allow us to detect carotenoid compounds. Therefore, we propose that future study should investigate the carotenoids content in $P$. mira fruit and correlate with our transcriptome data to illuminate the part played by carotenoids in fruit flesh coloration.

\section{Conclusions}

In the present investigation, we integrated transcriptome and metabolome analysis to clarify the regulatory network underlying fruit flesh coloration in Prunus mira. According to the transcriptome changes in the studied 3 fruit types during the various developmental stages, the determination of fruit flesh pigmentation mainly occurred during the fruit ripening period. We further identified a set of 563 differentially expressed genes associated with fruit flesh pigmentation and other major differentiations between the 3 fruit types. Metabolic profiling during the fruit ripening stage revealed 40 differential metabolites between the 3 fruit types, including 5 anthocyanins, which are suggested to be the key molecules associated with flesh coloration in P. mira. The regulatory network constructed based on the detected anthocyanin compounds and their correlated genes not only illuminated the regulatory mechanism underlying their biosynthesis but also provided a framework for understanding the interplay between transcriptional expression and metabolite levels according to the 
pigmentation in $P$. mira fruit flesh. The findings from this study will benefit molecular breeders in their effort to improve fruit appeal and quality in peach.

\section{Methods}

\section{Fruit materials}

Fruit of wild and semi-wild Prunus mira trees displaying different flesh colors: PMHR (blood-colored flesh; wild), PMHY (yellow-colored flesh; wild) and PMHF (milkwhite colored flesh; semi-wild) were harvested at the pithardening (60 days after bloom) (A), cell enlargement (85 days after bloom) (B) and fruit ripening (95 days after bloom) (C) stages (Fig. 1). All fruits were directly collected from the plants at the above mentioned developmental stages. PMHF is considered a good quality fruit by local farmers leading to a gradual increase of its cultivation. Fruits were randomly collected from 50 years-old individual wild trees and 10 years-old semi-wild tree in the city of Linzhi $\left(26^{\circ} 52^{\prime}-30^{\circ} 40^{\prime}\right.$ latitude, $92^{\circ} 09^{\prime}-98^{\circ} 47^{\prime}$, longitude), Nidi village in the southeast of the Tibet autonomous region (China). The collection of $P$. mira samples in the wild was authorized by the Tibet Academy of Agricultural Sciences through the permit number: $20171801016 \mathrm{pt}-1$. The formal identification of the species was conducted by the corresponding author of this article. Voucher specimens of the fruits are deposited to the germplasm collection bureau of the Institute of Vegetables, Tibet Academy of Agricultural and Animal Husbandry Sciences, under the codes: 2X51PM201729, 2X51PM201730 and 2X51PM201731. Then, the fruits were mixed and three biological replicates were sampled, representing in total 27 samples. Flesh samples of the fresh fruits were dissected using a blade, frozen immediately in liquid nitrogen in the field, transported to the laboratory and then stored at $-80^{\circ} \mathrm{C}$ until further use.

\section{Transcriptome sequencing}

Nine libraries based on samples representing the three fruit flesh colors and the three developmental stages were constructed for transcriptome sequencing. Total RNAs were extracted using TRIzol reagent (TaKaRa, Dalian, China) according to the manufacturer's instructions, and the mRNAs were enriched using magnetic oligo (dT) beads. The quantity and quality of mRNAs were assessed by ND-1000 Nanodrop spectrometer (Nanodrop Technologies, USA) and the Agilent 2100 Bioanalyzer (Agilent Technologies, USA). The mRNAs were added to a fragmentation buffer to break into short fragments. The cDNAs were synthesized with a six base random primer and then, AMPure XP beads were use to purify the double-stranded cDNA. The purified doublestranded cDNAs were first repaired at the end, a tail was added, the sequencing connector connected, and the fragment size was selected using the AMPure XP beads.
Finally, PCR amplification was performed and a chainspecific cDNA library was obtained by purifying PCR products with the AMPure XP beads. Sequencing was performed following standard Illumina methods and protocols. The cDNA libraries were sequenced on an Illumina Hiseq platform and $150 \mathrm{bp}$ single-end reads were generated at the Mega Genomics Co., Ltd. (www. megagenomics.cn).

\section{Transcriptome data analysis}

The sequencing reads containing low-quality (containing > $50 \%$ bases with a Phred quality score $<15$ ), more than $1 \%$ ambiguous residues $(\mathrm{N})$ and adaptor sequences were removed before downstream analyses using the FastQC tool (http://www.bioinformatics.babraham.ac.uk/projects/fastqc/ ). After filtering, the remaining high-quality reads "clean reads" stored in FASTQ were used for statistical analyses. The clean reads were then mapped to the $P$. persica reference genome (ftp://ftp.ncbi.nlm.nih.gov/genomes/all/GCF/ 000/346/465/GCF_000346465.2_Prunus_persica_NCBIv2/ GCF_000346465.2_Prunus_persica_NCBIv2_genomic.fna. gz) [27] using the TopHat2 tool [48]. The genomic localization results of all sequencing reads data were assembled with the cufflinks package [49], and then were compared to the known genetic models in order to identify new genes and alternative splicing events using the cuffcompare package [49]. We used the blast software [50] and the nr [51], swiss-prot [52], GO [53], KEGG [54] databases to obtain annotated information about the new genes. The gene expression level was determined according to the number of fragments per kilobase of exon per million fragments mapped (FPKM). The EBSeq program [55] was used to perform the differential expression analysis with fold change $(\mathrm{FC}) \geq 2$ or $\mathrm{FC} \leq 1 / 2$ and false discovery rate $(\mathrm{FDR})<0.01$ set as screening criteria. Gene ontology (GO) annotation and kyoto encyclopedia of genes and genomes (KEGG) pathway analyses were conducted using the Blast2GO software [56].

\section{Metabolic profiling}

The sample preparation, extract analysis, metabolite identification and quantification were performed at Wuhan MetWare Biotechnology Co., Ltd. (www.metware.cn) following their standard procedures and previously described by Wang et al. [57] and Cao et al. [58].

\section{Metabolite data analysis}

First, a quality control (QC) analysis was performed to verify the reliability of the data. Sample extracts were mixed and inserted into every 10 samples to monitor the changes in repeated analyses. Metabolomics data have the characteristics of high dimension dataset, so it is necessary to combine univariate and multivariate statistical analyses to accurately excavate the differential metabolites. Statistical analyses were performed using the 
Analyst 1.6.1 software (AB SCIEX, Ontario, Canada). Statistical significance and fold change of the metabolites between the samples were tested with two-paired $t$ test. The supervised multivariate method, partial least squares-discriminant analysis (PLS-DA), was used to maximize the metabolome difference between the developmental stages, as well as the difference between the three flesh colored fruits. The relative importance of each metabolite to the PLS-DA model was evaluated using the variable importance in projection (VIP). Metabolites with VIP $\geq 1$ and fold change $\geq 2$ or fold change $\leq 0.5$ were defined as differentially accumulated metabolites between compared samples $[57,58]$.

\section{Integrative analysis of metabolome and transcriptome datasets}

For the joint analysis between the metabolome and transcriptome datasets, the mean of all biological replicates of differential metabolites in the metabolome data and the mean value of expression of differential transcripts in the transcriptome data were calculated. Next, the $\log _{2}$ transformed datasets were loaded in the 'cor' package from the R software (www.r-project.org/). The Pearson correlation $(r)$ between metabolites and transcripts was represented by network diagrams, and the differential genes and metabolites between PMHF, PMHR and PMHY were selected when $R^{2}>0.9$ [46]. Metabolome and transcriptome relationships were visualized using the Cytoscape software version 3.6.1 [59].

\section{qRT-PCR expression profiling of selected genes}

RNAs from flesh samples were extracted using the EASYspin Plus kit (Aidlab Biotechnologies, China) according to the manufacturer's instructions. The RNA was treated with DNaseI and $1 \mu \mathrm{g}$ RNA was reverse transcribed with oligo (dT23) primer using the FastQuant RT kit (Tiangen Biotech, China) in a final volume of $25 \mu \mathrm{L}$. The specific primer pairs of the ten selected genes were designed using the Primer5.0 software [60] and presented in Additional file 18: Table S13. The qRT-PCR analysis was performed as described by Dossa et al. [61] using the ChamQ SYBR qPCR Master Mix (Vazyme Biotec, China) on a Light Cycler 480 II (Roche, Switzerland). Each reaction was performed using a $20 \mu \mathrm{L}$ mixture containing $10 \mu \mathrm{L}$ of $2 \times$ ChamQ SYBR qPCR Master Mix, $6 \mu \mathrm{L}$ of nuclease-free water, $1 \mu \mathrm{L}$ of each primer $(10 \mathrm{mM})$, and $2 \mu \mathrm{L}$ of 4 -fold diluted cDNA. All of the reactions were run in 96-well plates and each cDNA was analyzed in triplicate. The following cycling profile used: $95^{\circ} \mathrm{C}$ for $30 \mathrm{~s}$, followed by 40 cycles of $95^{\circ} \mathrm{C} / 10 \mathrm{~s}, 60^{\circ} \mathrm{C} / 30 \mathrm{~s}$. The relative expression levels of the selected genes were normalized to the expression level of the ACTIN gene [62]. This analysis was carried out in three independent biological replicates and three technical replicates of each biological replicate.

\section{Supplementary information}

Supplementary information accompanies this paper at https://doi.org/10. 1186/s12870-019-2074-6.

Additional file 1: Table S1. Overview of the transcriptome sequencing dataset and quality check in 3 Prunus mira fruit types (PMHF, PMHR and $\mathrm{PMHY}$ ) during 3 developmental stages (pit-hardening (A), cell

enlargement (B) and fruit ripening (C)). (XLSX $10 \mathrm{~kb}$ )

Additional file 2: Table S2. Full list of the unique genes detected in Prunus mira fruit transcriptome and their functional annotation. (XLSX 2092 kb)

Additional file 3: Table S3. List of the novel genes specific to Prunus mira detected from fruit transcriptome and their functional annotation. (XLSX 109 kb)

Additional file 4: Table S4. List of the optimized genes based on gene structure optimization analysis in P. persica. (XLSX $301 \mathrm{~kb}$ )

Additional file 5: Table S5. Intron-exon structure of the optimized genes in P. persica. (XLSX $3036 \mathrm{~kb}$ )

Additional file 6: Table S6. List of all the differentially expressed genes identified in this study along with their FPKM data. (XLSX 4901 kb)

Additional file 7: Figure S1. KEGG enrichment analysis of the 343 genes constantly and differentially expressed during the fruit development and ripening in Prunus mira. (TIF $522 \mathrm{~kb}$ )

Additional file 8: Figure S2. KEGG enrichment analysis of the 3736 genes constantly and differentially expressed during the transition from the pit-hardening to the cell enlargement stages in Prunus mira. (TIF 565 kb)

Additional file 9: Figure S3. KEGG enrichment analysis of the 607 genes constantly and differentially expressed during the transition from the cell enlargement to the fruit ripening stages in Prunus mira. (TIF $556 \mathrm{~kb}$ )

Additional file 10: Figure S4. qRT-PCR $\left(2^{-\Delta \Delta c t}\right)$ analysis of 10 selected genes within the differentially expressed genes detected in this study. Correlation analysis between qRT-PCR and RNA-seq (log2fold change). (TIF $886 \mathrm{~kb}$ )

Additional file 11: Table S7. List of the differentially expressed genes between the 3 Prunus mira fruit types (PMHF, PMHR and PMHY) representing the 'core transcriptome' and their functional annotation. (XLSX 26 kb)

Additional file 12: Figure S5. KEGG enrichment analysis of the 563 genes differentially expressed in PMHF, PMHR and PMHY during the fruit ripening stage, representing the 'core transcriptome' in Prunus mira.s (TIF $522 \mathrm{~kb}$ )

Additional file 13: Table S8. List of the differentially expressed genes between the 3 Prunus mira fruit types (PMHF, PMHR and PMHY) mapped to the terpernoid-carotenoid pathways and phenylpropanoid-flavonoid pathways and their expression level during the fruit ripening stage (C). (XLSX $11 \mathrm{~kb}$ )

Additional file 14: Table S9. List of the $b H L H$ and MYB transcription factors detected in the 'core transcriptome' and their expression fold change in group comparisons between the 3 Prunus mira fruit types (PMHF, PMHR and PMHY) during the fruit ripening stage. (XLSX $10 \mathrm{~kb}$ )

Additional file 15: Table S10. Overview of the metabolites detected and quantified in 3 fruit types of Prunus mira (PMHF, PMHR and PMHY) during 3 developmental stages (pit-hardening (A), cell enlargement (B) and fruit ripening $(C)$ ). Data are from 3 biological replicates and the mix01 to mix05 represent the mixture of sample extracts. (XLSX $138 \mathrm{~kb}$ )

Additional file 16: Table S11. List of the differential accumulated metabolites between the 3 Prunus mira fruit types (PMHF, PMHR and $\mathrm{PMHY}$ ) representing the 'core metabolome' and their expression fold 
change between group comparisons during the fruit ripening stage. (XLSX $15 \mathrm{~kb}$ )

Additional file 17: Table S12. List of genes showing a high correlation $\left(\mathrm{R}^{2}>0.9\right)$ with the 5 anthocyanins detected as important molecules involved in the fruit flesh coloration in Prunus mira. (XLSX $68 \mathrm{~kb}$ )

Additional file 18: Table S13. The primer sequences for real time PCR. (XLSX 9 kb)

\section{Abbreviations}

4CL: 4 coumarate CoA ligase; ANS: Anthocyanidin synthase; bHLH: basic helixloop-helix; C4H: Cinnamic acid 4-hydroxylase; CHI: Chalcone isomerase; CHS: Chalcone synthase; DAM: Differentially accumulated metabolites; DEG: Differentially expressed genes; DFR: Dihydroflavonol 4-reductase; DHK: Dihydro-kaempferol; F3H: Flavonone 3-hydroxylase; FC: Fold change; FPKM: Fragments per kilobase of exon per million fragments mapped; GO: Gene ontology; HCA: Hierarchical cluster analysis; KEGG: Kyoto encyclopedia of genes and genome; LDOX: Leucoanthocyanidin dioxygenase; PAL: Phenylalanine ammonia-lyase; PCA: Principal component analysis; PLS-DA: Partial least squares-discriminant analysis; POD: Peroxidase; QC: Quality control; UFGT: UDP-glucose-flavonoid 3-O-glucosyltransferase; VIP: Variable importance in projection

\section{Acknowledgements}

Not applicable.

\section{Author's contributions}

$H Y, F Z, Y C$ and $X Z$ conceived, designed and supervised the experiment; $H Y$ and JS wrote the manuscript; HY, JS, SZ, GP and SW performed the experiment; JS and FZ provided support in lab experiment and data analysis. $\mathrm{HY}$ and JS, analyzed the data. All authors read and approved the manuscript.

\section{Funding}

This work was funded by the Financial Special Fund; grant number [XZNKY 2018-C-0025] and the Tibet Department of Major Projects. The funders had no role in the experimental design, data collection and analysis or writing the manuscript.

\section{Availability of data and materials}

All data generated or analysed during this study are included in this published article and its supplementary information files. The raw RNA-seq data are freely available at: www.ncbi.nlm.nih.gov/bioproject/PRJNA510449. The annotated gene file and files containing the gene sequences in fasta are available at Figshare: https://figshare.com/articles/Prunus_mira_gene_annotation_file/9598799 and https://figshare.com/articles/Prunus_mira_genes_in_ fasta format/9598808. File containing the gene sequences in fasta has also been deposited at DDBJ/EMBL/GenBank under the accession GHVK00000000. The version described in this paper is the first version, GHVK01000000.

\section{Ethics approval and consent to participate}

Not applicable.

\section{Consent for publication}

Not applicable.

\section{Competing interests}

The authors declare that they have no competing interests.

\section{Author details}

${ }^{1}$ The ministry of agriculture of Qinghai-Tibet plateau fruit trees scientific observation test station, Lhasa 850032, Tibet, China. Institute of Vegetables, Tibet Academy of Agricultural and Animal Husbandry Sciences, Lhasa 850002, Tibet, China. ${ }^{3}$ Wuhan Metware Biotechnology Co., Ltd, Wuhan 430070, China.

Received: 11 January 2019 Accepted: 14 October 2019

Published online: 01 November 2019

\section{References}

1. Dong GZ. The investigation of Prunus mira Koehne in Tibet. Q For By-prod Spec China. 1991;3:44-5.
2. Tian Y, Xing C, Cao Y, Wang C, Guan F, Li R, Meng F. Evaluation of genetic diversity on Prunus mira Koehne by using ISSR and RAPD markers. Biotechnol Biotechnol Equip. 2015;29:1053-61.

3. Wang ZH, Zhuang EJ. Fruit annals in China _ scroll of peach and nectarine. Beijing China, Forestry Publishing House 2001.

4. Huang $H$, Cheng Z, Zhang Z, Wang Y. History of cultivation and trends in China. In: Layne DR, Bassi D, editors. The peach: botany production and uses. Wallingford: CABl; 2008. p. 37-60.

5. Guan F, Wang S, Li R, Peng M, Meng F. Genetic diversity of wild peach (Prunus mira Koehne kov et kpst) from different altitudes in the Tibetan plateau by pollen morphous and RAPD markers. Hortscience. 2014;49:1017-22.

6. Peng M, Guan FC, Tao L, Li RQ, Wang C, Meng FJ. Analysis of genetic relationship on Amygdalus mira (Koehne) Ricker with other peach species using simple sequence repeat (SSR). Biochem System Ecol. 2015;62:98-105.

7. Gil MI, Tomas-Barberan FA, Hess-Pierce B, Kader AA. Antioxidant capacities phenolic compounds carotenoids and vitamin C contents of nectarine peach and plum cultivars from California. J Agric Food Chem. 2002;50:4976-82.

8. Tuan PA, Bai S, Yaegaki H, Tamura T, Hihara S, Moriguchi T, Oda K. The crucial role of PpMYB101 in anthocyanin accumulation in peach and relationships between its allelic type and skin color phenotype. BMC Plant Biol. 2015;15:280.

9. Bao W, Wuyun T, Li T, Liu H, Jiang Z, Zhu X, Du H, Bai Y. Genetic diversity and population structure of Prunus mira (Koehne) from the Tibet plateau in China and recommended conservation strategies. PLoS One. 2017;12:e0188685.

10. Illa E, Eduardo I, Audergon JM, Barale FE, Dirlewanger Li X, Moing A, Lambert P, Dantec LL, Gao Z, Poessel JL, Pozzi C, Rossini L, Vecchietti A, Arus $P$, Howad W. Saturating the Prunus (stone fruits) genome with candidate genes for fruit quality. Mol Breed. 2011;28:667-82.

11. Chen C. Overview of plant pigments. In: Chen C (eds) Pigments in fruits and vegetables. Springer New York NY 2015 1-7.

12. Jaakola L. New insights into the regulation of anthocyanin biosynthesis in fruits. Trends Plant Sci. 2013;18:477-83.

13. Morita Y, Saitoh M, Hoshino A, Nitasaka E, lida S. Isolation of CDNAs for R2R3-MYB bHLH and WDR transcriptional regulators and identification of $\mathrm{C}$ and ca mutations conferring white flowers in the Japanese morning glory. Plant Cell Physiol. 2006:4:457-70.

14. Allan AC, Hellens RP, Laing WA. MYB transcription factors that colour our fruit. Trends Plant Sci. 2008;13:99-102.

15. Fu X, Cheng S, Liao Y, Huang B, Du B, Zeng W, Jiang Y, Duan X, Yang Z. Comparative analysis of pigments in red and yellow banana fruit. Food Chem. 2018;239:1009-18.

16. Ruiz-Sola MA, Rodriguez-Concepcion M. Carotenoid biosynthesis in Arabidopsis: a colorful pathway. Arabidopsis Book. 2012;10:e0158.

17. Tsuda T, Yamaguchi M, Honda C, Moriguchi T. Expression of anthocyanin biosynthetic genes in the skin of peach and nectarine fruit. J Amer Soc Hort Sci. 2004;129:857-62.

18. Brandi F, Bar E, Mourgues F, Horváth G, Turcsi E, Giuliano G, Livera-ni A, Tartarini S, Lewinsohn E, Rosati C. Study of 'Redhaven' and its white-fleshed mutant suggests a key role of CCD4 carotenoid dioxygenase in carotenoid and norisoprenoid volatile metabolism. BMC Plant Biol. 2011;11:24.

19. Falchi R, Vendramin E, Zanon L, Scalabrin S, Cipriani G, Verde I, Vizzotto G. Morgante $M$ three distinct mutational mechanisms acting on a single gene underpin the origin of yellow flesh in peach. Plant J. 2013;7:6175-87.

20. Jiao Y, Ma RJ, Shen ZJ, Yan J, Yu ML. Gene regulation of anthocyanin biosynthesis in two blood-flesh peach (Prunus persica $(L)$ Batsch) cultivars during fruit development. J Zhejiang Univ Scie B. 2014;15:809-19.

21. Yan J, Cai Z, Shen Z, Ma R, Yu M. Proanthocyanidin monomers and cyanidin 3-o-glucoside accumulation in blood-flesh peach (Prunus persica (I) Batsch) fruit. Arch Biol Sci. 2017;69:611-7.

22. Li Y, Fang J, Qi X, Lin M, Zhong Y, Sun L, Cui W. Combined analysis of the fruit metabolome and transcriptome reveals candidate genes involved in flavonoid biosynthesis in Actinidia arguta. Int J Mol Sci. 2018;19:1471.

23. Lou Q, Liu Y, Qi Y, Jiao S, Tian F, Jiang L, Wang Y. Transcriptome sequencing and metabolite analysis reveals the role of delphinidin metabolism in flower colour in grape hyacinth. J Exp Bot. 2014;65:57-3164.

24. Matus JT. Transcriptomic and metabolomic networks in the grape berry illustrate that it takes more than flavonoids to fight against ultraviolet radiation. Front Plant Sci. 2016;7:1337.

25. Wang Z, Du H, Zhai R, Song L, Ma F, Xu L. Transcriptome analysis reveals candidate genes related to color fading of 'red Bartlett' (Pyrus communis L). Front Plant Sci. 2017;8:455. 
26. Wang Z, Cui Y, Vainstein A, Chen S, Ma H. Regulation of fig (Ficus carica L) fruit color: metabolomic and transcriptomic analyses of the flavonoid biosynthetic pathway. Front Plant Sci. 2017;8:1990.

27. The International Peach Genome Initiative. The high-quality draft genome of peach (Prunus persica) identifies unique patterns of genetic diversity domestication and genome evolution. Nat Genet. 2013:45:487-94.

28. Li XW, Meng XQ, Jia HJ, Yu ML, Ma RJ, Wang LR, Cao K, Shen ZJ, Niu L, Tian JB, Chen MJ, Xie M, Arus P, Gao ZS, Aranzana MJ. Peach genetic resources: diversity population structure and linkage disequilibrium. BMC Genet. 2013;14:84

29. Lombardo VA, Osorio S, Borsani J, Lauxmann MA, Bustamante CA, Budde CO, Andreo CS, Lara MV, Fernie AR. Drincovich MF metabolic profiling during peach fruit development and ripening reveals the metabolic networks that underpin each developmental stage. Plant Physiol. 2011;157:1696-710.

30. Chalmers DJ, van den Ende B. A reappraisal of the growth and development of peach fruit. Aust J Plant Physiol. 1975;2:623-34.

31. Roca M, Mínguez-Mosquera Ml. Carotenoid levels during the period of growth and ripening in fruits of different olive varieties (Hojiblanca, Picual and Arbequina). J Plant Physiol. 2003;160:451-9.

32. Cao K, Ding T, Mao D, Zhu G, Fang W, Chen C, Wang X, Wang L. Transcriptome analysis reveals novel genes involved in anthocyanin biosynthesis in the flesh of peach. Plant Physiol Biochem. 2018;123:94-102.

33. Rahim MA, Busatto N, Trainotti L. Regulation of anthocyanin biosynthesis in peach fruits. Planta. 2014;240:913.

34. Dixon RA, Steele CL. Flavonoids and isoflavonoids - a gold mine for metabolic engineering. Trends Plant Sci. 1999;4:394-400.

35. Takos AM, Jaffé FW, Jacob SR, Bogs J, Robinson SP, Walker AR. Lightinduced expression of a MYB gene regulates anthocyanin biosynthesis in red apples. Plant Physiol. 2006;142:1216-32.

36. Ravaglia D, Espley R, Henry-Kirk R, Andreotti C, Ziosi V, Hellens RP, Costa G. Allan AC transcriptional regulation of flavonoid biosynthesis in nectarine (Prunus persica) by a set of R2R3 MYB transcription factors. BMC Plant Biol. 2013;13:68.

37. Zhang J, Pan H, Gao Z, Shu B, Qi Y, Yi X, Qin G, Sheng Y, Chen H, Xu Y. Transcriptome analysis of colouration related genes in two white-fleshed nectarine varieties and their yellow-fleshed mutants. Biotechnol Biotechnol Equip. 2018. https://doi.org/10.1080/1310281820181438208.

38. Chen Y, Mao Y, Liu H, Yu F, Li S, Yin T. Transcriptome analysis of differentially expressed genes relevant to variegation in peach flowers. PLoS One. 2014;9:e90842.

39. Zhao Y, Dong W, Wang K, Zhang B, Allan AC, Lin-Wang K, Chen K. Xu C differential sensitivity of fruit pigmentation to ultraviolet light between two peach cultivars. Front Plant Sci. 2017:8:1552.

40. Wu X, Gong Q, Ni X, Zhou Y, Gao Z. UFGT: the key enzyme associated with the petals variegation in Japanese apricot. Front Plant Sci. 2017;8:108.

41. To KY, Wang CK. Molecular breeding of flower color. In: floriculture ornamental and plant biotechnology: advances and topical issues volume I edited by Teixeira da Silva JA, Isleworth, England, 2006, 300-310.

42. Zhang Z, Pang $X$, Xuewu D, Ji Z, Jiang Y. Role of peroxidase in anthocyanin degradation in litchi fruit pericarp. Food Chem. 2005;90:47-52.

43. Zipor G, Duarte P, Carqueijeiro I, Shahar L, Ovadia R, Teper-Bamnolker P, Eshel D, Levin Y, Doron-Faigenboim A, Sottomayor M, Oren-Shamir M. In planta anthocyanin degradation by a vacuolar class III peroxidase in Brunfelsia calycina flowers. New Phytol. 2015;205:653-65.

44. Niu J, Zhang G, Zhang W, Goltsev V, Sun S, Wang J, Li P, Ma F. Anthocyanin concentration depends on the counterbalance between its synthesis and degradation in plum fruit at high temperature. Sci Rep. 2017;7:7684.

45. Movahed N, Pastore C, Cellini A, Allegro G, Valentini G, Zenoni S, Cavallini E, D'Incà E, Tornielli GB, Filippetti I. The grapevine VviPrx31 peroxidase as a candidate gene involved in anthocyanin degradation in ripening berries under high temperature. J Plant Res. 2016;129:513-26.

46. Cho K, Cho K, Sohn H, Ha IJ, Hong S, Lee H, Kim Y, Nam MH. Network analysis of the metabolome and transcriptome reveals novel regulation of potato pigmentation. J Exp Bot. 2016;67:1519-33.

47. Miyazawa T, Nakagawa K, Kudo M, Muraishi K, Someva K. Direct intestinal absorption of red fruit anthocyanins cyanidin-3-glucoside and cyanidin-35diglucoside into rats and humans. J Agric Food Chem. 1999;47:1083-91.

48. Kim D, Pertea G, Trapnell C, Pimentel H, Kelley R, Salzberg SL. TopHat2: accurate alignment of transcriptomes in the presence of insertions deletions and gene fusions. Genome Biol. 2013;14:R36.
49. Trapnell C, Williams BA, Pertea G, Mortazavi A, Kwan G, van Baren MJ, Salzberg SL, Wold BJ, Pachter L. Transcript assembly and quantification by RNA-Seq reveals unannotated transcripts and isoform switching during cell differentiation. Nat Biotechnol. 2010;28:511-5.

50. Altschul SF, Madden TL, Schäffer AA, Zhang Z, Zhang J, Miller W, Lipman DJ. Gapped BLAST and PSI BLAST: A new generation of protein database search programs. Nucleic Acids Res. 1997;25:3389-402.

51. Deng Y, Li J, Wu S, Zhu Y, Chen Y, He F. Integrated NR database in protein annotation system and its localization. Comput Eng. 2006;32:71-4.

52. Apweiler R, Bairoch A, Wu CH, Barker W, Boeckmann B, Ferro S, Gasteiger E, Huang H, Lopez R, Magrane M, Martin MJ, Natale DA, O'Donovan C, Redaschi N, Yeh LS. UniProt: the universal protein knowledgebase. Nucleic Acids Res. 2004;32:D115-9.

53. Shburner M, Bal CA, Blake JA, Botstein D, Butler H, Cherry JM, Davis AP, Dolinski K, Dwight SS, Eppig JT, Harris MA, Hill DP, Issel-Tarver L, Kasarskis A, Lewis S, Matese JC, Richardson JE, Ringwald M, Rubin GM, Sherlock G. Gene ontology: tool for the unification of biology. Nat Genet. 2000;25:25-9.

54. Kanehisa M, Goto S, Kawashima S, Okuno Y, Hattori M. The KEGG resource for deciphering the genome. Nucleic Acids Res. 2004;32:D277-80.

55. Leng N, Dawson JA, Thomson JA, Ruotti V, Rissman Al, Smits BMG, Haag JD, Gould MN, Stewart RM, Kendziorski C. EBSeq: an empirical bayes hierarchical model for inference in RNA-seq experiments. Bioinformatics. 2013;29:1035-43.

56. Conesa BA, Götz S, García-Gómez J, Terol J, Talón M, Robles M. Blast2GO: a universal tool for annotation visualization and analysis in functional genomics research. Bioinformatics. 2005;21:3674-6.

57. Wang Y, Zeng X, Xu Q, Mei X, Yuan H, Jiabu D, Sang Z, Nyima T. Metabolite profiling in two contrasting Tibetan hulless barley cultivars revealed the core salt-responsive metabolome and key salt-tolerance biomarkers. AoB Plants, 2019;11:plz021.

58. Cao H, Ji Y, Li S, Lu L, Tian M, Yang W, Li H. Extensive metabolic profiles of leaves and stems from the medicinal plant Dendrobium officinale Kimura et Migo. Metabolites. 2019;9:215.

59. Su G, Morris JH, Demchak B, Bader GD. Biological network exploration with Cytoscape 3. Curr Protoc Bioinformatics. 2014;47:1-24.

60. Lalitha S. Primer premier 5. Biotechnol Softw Internet Rep. 2000;1:270-2.

61. Dossa K, Mmadi MA, Zhou R, Zhou Q, Yang M, Cisse N, Diouf D, Wang L, Zhang $X$. The contrasting response to drought and waterlogging is underpinned by divergent DNA methylation programs associated with transcript accumulation in sesame. Plant Sci. 2018;277:207-17.

62. Cao Y, Luo Q, Tian Y, Meng F. Physiological and proteomic analyses of the drought stress response in Amygdalus mira (Koehne) Yü et Lu roots. BMC Plant Biol. 2017;17:53.

\section{Publisher's Note}

Springer Nature remains neutral with regard to jurisdictional claims in published maps and institutional affiliations.

Ready to submit your research? Choose BMC and benefit from:

- fast, convenient online submission

- thorough peer review by experienced researchers in your field

- rapid publication on acceptance

- support for research data, including large and complex data types

- gold Open Access which fosters wider collaboration and increased citations

- maximum visibility for your research: over $100 \mathrm{M}$ website views per year

At BMC, research is always in progress.

Learn more biomedcentral.com/submissions 